\title{
ARTICLE OPEN \\ A metabolomic study of the effect of Candida albicans glutamate dehydrogenase deletion on growth and morphogenesis
}

\author{
Ting-Li Han (iD ${ }^{1,2}$, Richard D. Cannon (iD ${ }^{3}$, Sandra M. Gallo ${ }^{1}$ and Silas G. Villas-Bôas ${ }^{1}$
}

There are two glutamate dehydrogenases in the pathogenic fungus Candida albicans. One is an NAD ${ }^{+}$-dependent glutamate dehydrogenase $(\mathrm{GDH} 2)$ and the other is an NADPH-dependent glutamate dehydrogenase (GDH3). These two enzymes are part of the nitrogen and nicotinate/nicotinamide metabolic pathways, which have been identified in our previous studies as potentially playing an important role in C. albicans morphogenesis. In this study, we created single gene knockout mutants of both dehydrogenases in order to investigate whether or not they affect the morphogenesis of $C$. albicans. The GDH genes were deleted and the phenotypes of the knockout mutants were studied by growth characterisation, metabolomics, isotope labelling experiments, and by quantifying cofactors under various hyphae-inducing conditions. We found that the gdh2/gdh2 mutant was unable to grow on either arginine or proline as a sole carbon and nitrogen source. While the gdh3/gdh3 mutant could grow on these carbon and nitrogen sources, the strain was locked in the yeast morphology in proline-containing medium. We detected different concentrations of $\mathrm{ATP}_{\mathrm{T}} \mathrm{NAD}^{+}, \mathrm{NADH}, \mathrm{NAPD}{ }^{+}, \mathrm{NADPH}$, as well as 62 other metabolites, and 19 isotopically labelled metabolites between the mutant and the wild-type strains. These differences were associated with 44 known metabolic pathways. It appears that the disequilibrium of cofactors in the $g d h 3 / g d h 3$ mutant leads to characteristic proline degradation in the central carbon metabolism. The analysis of the $g d h 2 / g d h 2$ and the $g d h 3 / g d h 3$ mutants confirmed our hypothesis that redox potential and nitrogen metabolism are related to filament formation and identified these metabolic pathways as potential drug targets to inhibit morphogenesis.

npj Biofilms and Microbiomes (2019)5:13; https://doi.org/10.1038/s41522-019-0086-5

\section{INTRODUCTION}

Candida albicans is a polymorphic fungus that grows on various human mucosal surfaces. The morphological switch from budding yeast to filamentous forms is often associated with its biological adaptation as an opportunistic pathogen of humans. The yeast-tohyphal transition has been previously reported to play an important role in C. albicans systemic infections by enabling the hyphae to penetrate endothelial tissue and subsequently seed the bloodstream with yeast cells ${ }^{1-3}$. C. albicans yeast cells are also capable of surviving during phagocytosis by triggering filamentous growth and eventually bursting out of the macrophage or neutrophil ${ }^{4,5}$. Therefore, understanding the cellular mechanisms that drive morphogenesis is crucial in defining its pathogenic traits.

In our previous studies ${ }^{6-8}$, we have demonstrated that during the yeast-to-hyphal transition the central carbon metabolism of $C$. albicans is globally downregulated. In particular, the pathways involved in the metabolism of alanine, $\beta$-alanine, aspartate, cysteine, histidine, glutamate, methionine, nitrogen, and nicotinate/nicotinamide, as well as the biosynthesis of acetyl-CoA are repressed. By using an isotope labelling experiment to trace the catabolism of the quorum sensing molecule phenylethyl alcohol, that represses hyphae formation under hyphae-inducing conditions, we have also found strong evidence for the involvement of
$\mathrm{NADP}^{+} / \mathrm{NADPH}$ and $\mathrm{NAD}^{+} / \mathrm{NADH}$ in phenylethyl alcohol-mediated morphogenesis. This indicates that the redox balance of the fungal cells could be intrinsically associated with morphogenesis. Therefore, we decided to knock out the genes encoding NAD ${ }^{+}$. dependent glutamate dehydrogenase $(G D H 2)$ and $\mathrm{NADPH}$ dependent glutamate dehydrogenase $(G D H 3)$ in order to assess the role of the cell's redox balance and nitrogen metabolism in C. albicans morphogenesis.

$G D H 2$ and $G D H 3$ play central roles in nitrogen assimilation and excretion (Fig. 1), and in the maintenance of the redox balance of the cell ${ }^{9-11}$. Although the reactions catalysed by Gdh2 and Gdh3 are reversible, the Gdh2-catalysed reaction favours the deamination of glutamate-its catabolism to $a$-ketoglutarate and ammonium $^{12}$. This reaction also generates $\mathrm{NADH}^{13}$. It is considered to be the final step in eukaryotic nitrogen excretion and very important in recycling oxidised $\mathrm{NAD}^{+}$. On the other hand, Gdh3 is predominantly involved in an anabolic reaction whereby inorganic ammonium is combined with $a$-ketoglutarate to form glutamate and, in the process, oxidises NADPH. Glutamate subsequently serves as an important nitrogen carrier for the biosynthesis of other amino acids via transamination reactions. In addition, dimorphic fungi such as Benjaminiella poitrasii, Mucor racemosus, and Schizophyllum commune have been reported to possess different $\mathrm{NAD}^{+}$- and $\mathrm{NADPH}-$ glutamate dehydrogenase activities

\footnotetext{
${ }^{1}$ School of Biological Sciences, The University of Auckland, Auckland, New Zealand; ${ }^{2}$ Department of Obstetrics and Gynecology, The First Affiliated Hospital of Chongqing Medical University, Chongqing, China and ${ }^{3}$ Sir John Walsh Research Institute, University of Otago Faculty of Dentistry, Dunedin, New Zealand

Correspondence: Ting-Li Han (t.han@auckland.ac.nz) or Silas G. Villas-Bôas (s.villas-boas@auckland.ac.nz)
}

Received: 20 December 2018 Accepted: 12 March 2019

Published online: 08 April 2019 


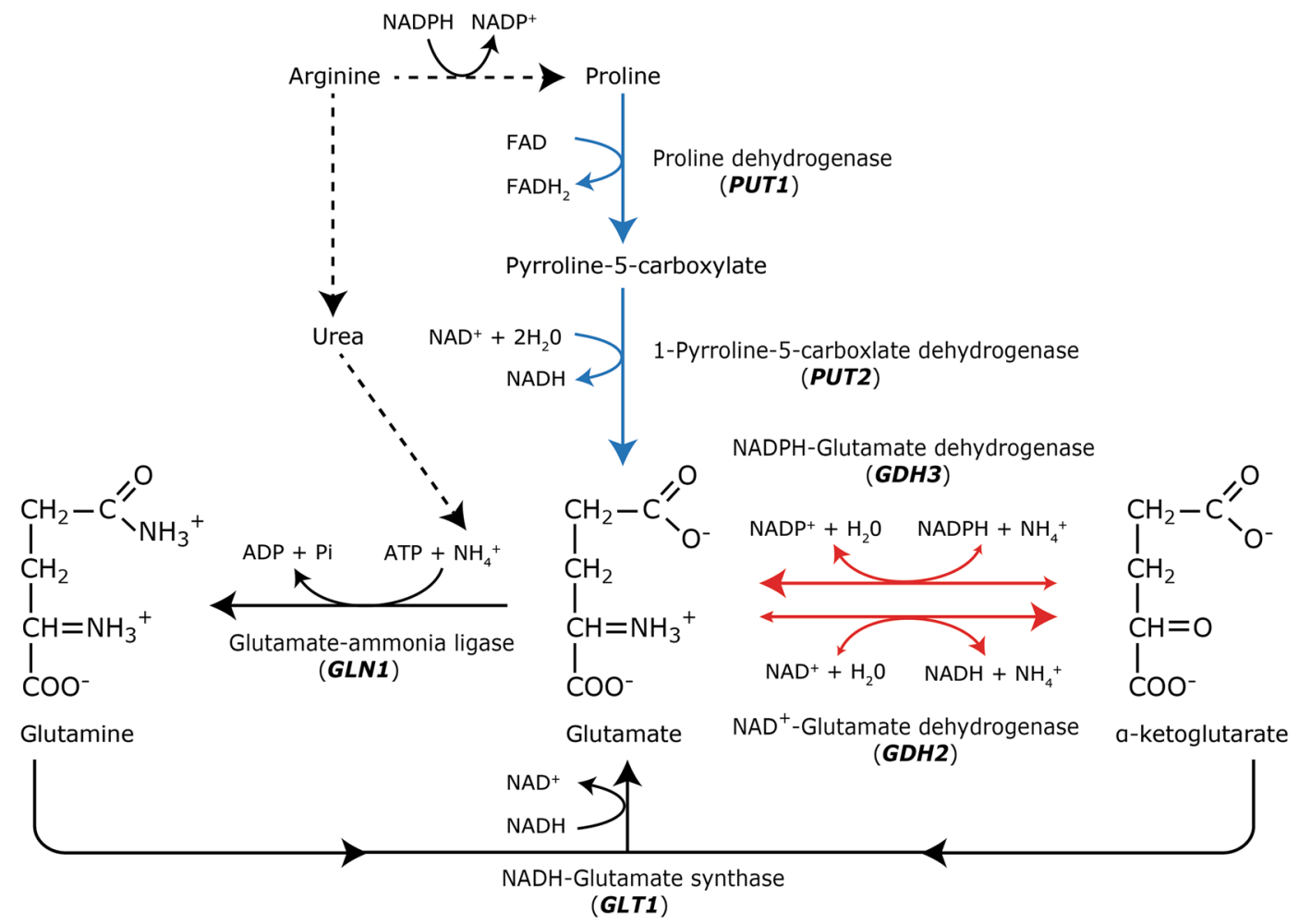

Fig. 1 Three primary metabolic pathways for nitrogen assimilation and glutamate metabolism in C. albicans. Red lines: the reactions catalysed by glutamate dehydrogenases $(G D H 2$ and $G D H 3)$. Large arrows indicate reactions that are more favourable, thermodynamically. Black lines: the reactions catalysed by a combination of GLN1 and GLT1, also referred to as GS and GOGAT in S. cerevisiae, respectively. Blue lines: the reactions catalysed by proline utilisation enzymes (PUT1 and PUT2). Black dashed line: the reactions responsible for arginine degradation to proline and ammonia

when growing in the yeast and filamentous forms ${ }^{14-16}$. In addition, there are two alternative pathways involved in nitrogen assimilation in yeast cells (Fig. 1). The second pathway is mediated by the combined enzymatic activities of glutamate-ammonia ligase (often called glutamine synthase in Saccharomyces cerevisiae) and glutamate synthase, encoded by GLN1 and GLT1 in C. albicans, and known as GS and GOGAT in S. cerevisiae, respectively ${ }^{17}$. GLN1 is predicted to catalyse the amination of glutamate to form glutamine. GLT1 then transfers the amide group from glutamine to a-ketoglutarate, synthesising two molecules of glutamate ${ }^{18}$. The third pathway is proline utilisation which occurs in mitochondria where proline is oxidised to pyrroline-5-carboxylate by mitochondrial proline dehydrogenase (PUT1) which, in turn, is further oxidised to glutamate by 1pyrroline-5-caboxylate dehydrogenase $(P U T 2)^{19}$. Although these two pathways have not been studied in C. albicans directly, they are likely to be present because orthologs of the genes encoding the $S$. cerevisiae enzymes have been found in the $C$. albicans genome (http://www.candidagenome.org//).

In this study, we used the SAT1 flipper method ${ }^{20}$ to knock out the $G D H 2$ or $G D H 3$ gene in C. albicans followed by characterisation of growth under hyphae-inducing conditions. To further characterise the effect of the $G D H 2$ and $G D H 3$ gene deletions on $C$. albicans metabolism under hyphae inducing conditions, we combined metabolomics and isotope labelling with targeted quantification of nucleotides (e.g. ATP, NAD ${ }^{+}, \mathrm{NADH}, \mathrm{NADP}^{+}$, and NAPDH). Identifying metabolic changes specific to the deletion of $G D H 2$ and $G D H 3$ genes will assist in the characterisation of the role of glutamate dehydrogenase in C. albicans morphogenesis, and hence its contribution to the virulence of this opportunistic pathogen.

\section{RESULTS}

PCR verification of GDH2/GDH3 genes knockout mutagenesis

The SAT1 flipper disruption cassettes for $G D H 2$ and $G D H 3$ gene deletions were generated successfully by PCR. Portions upstream (443 bp) and downstream (338 bp) of the GDH2 gene were amplified and fused with the $5269 \mathrm{bp}$ SAT1 fragment to form a disruption cassette ( $6 \mathrm{~kb})$ for $G D H 2$ gene deletion (Supplementary Fig. 2). Likewise, $527 \mathrm{bp}$ of the upstream and $446 \mathrm{bp}$ of the downstream regions of the $G D H 3$ gene were amplified and fused with the SAT1 fragment to form a disruption cassette ( $6 \mathrm{~kb})$ for $G D H 3$ gene deletion (Supplementary Fig. 2).

To confirm that the SAT1 flipper disruption cassettes had correctly replaced either the $G D H 2$ or $G D H 3$ gene after double crossover integrative transformation, the $5^{\prime}$ and $3^{\prime}$ junctions of disrupted alleles were PCR amplified. The successful allele disruptions were validated by PCR using primers $\mathrm{C} 1, \mathrm{C} 2, \mathrm{C} 3$, and C4 (Supplementary Fig. 1E) which generated the correct band sizes as illustrated in Supplementary Fig. 3. The PCR amplicon sizes for the $5^{\prime}$ check and $3^{\prime}$ check of GDH2 gene disruption, 490 and $980 \mathrm{bp}$, matched the predicted values of 466 and $950 \mathrm{bp}$, respectively. Furthermore, the PCR amplicon sizes for the $5^{\prime}$ check and $3^{\prime}$ check of GDH3 gene disruption, 600 and $790 \mathrm{bp}$, matched the predicted values of 607 and $751 \mathrm{bp}$, respectively.

To verify that the SAT1 disruption cassettes were successfully excised from GDH2/gdh2 and GDH3/gdh3 strains, genomic PCR was used with primers $\mathrm{C} 1$ and $\mathrm{C} 4$ to amplify the region between the upper end of $5^{\prime}$ flank sequences to the lower end of $3^{\prime}$ flank sequences to make sure that both alleles of each gene were removed (Supplementary Fig. 4). The expected PCR amplicon sizes after the excision of disruption cassettes from $G D H 2$ and $G D H 3$ gene deletions were 1147 and $1191 \mathrm{bp}$, respectively. Both PCR amplifications of these regions yielded bands of $\sim 1200 \mathrm{bp}$. These 


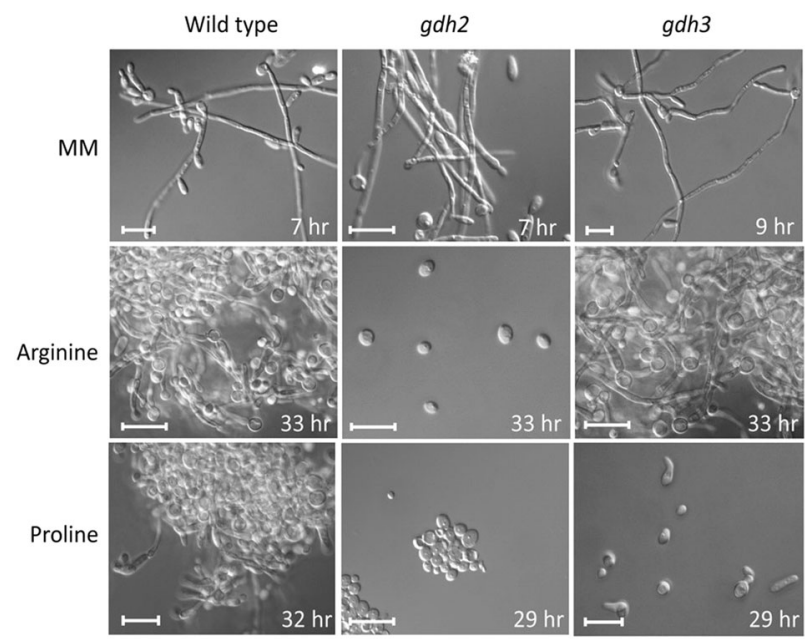

Fig. 2 The morphologies of the $g d h 2 / g d h 2$, gdh3/gdh3, and wildtype strains of $C$. albicans grown on various media. Cells were incubated at $37^{\circ} \mathrm{C}$ and examined at the end of exponential growth phase. MM medium is minimum mineral medium with $1 \%$ glucose. Arginine or proline media are minimum mineral medium without any carbon and nitrogen source but supplemented with either arginine or proline. The wild-type strain is C. albicans SC5314. The images were obtained by Nomarksi contrast microscopy with $\times 100$ magnification. The scale bars on each panel represent $10 \mu \mathrm{m}$

$P C R$ reactions verified the excision of the $S A T 1$ disruption cassette. Thus, we confirmed correct construction of the GDH2 and GDH3 knockout strains.

The morphologies of $g d h 2 / g d h 2$ and gdh3/gdh3 mutant strains, and the parental strain, grown on different media

There was no obvious difference in hyphal development between the wild-type (parental) and the mutant $C$. albicans strains when cultured in minimum mineral (MM) medium (Fig. 2), or under other hyphae-inducing conditions (Supplementary Fig. 7). However, when proline or arginine was provided as sole carbon and nitrogen sources, distinct differences were seen. In proline medium, the $g d h 3 / g d h 3$ mutant strain failed to form filaments, whilst the wild-type developed hyphae (Fig. 2). In contrast, in arginine medium both the gdh3/gdh3 and wild-type strains completely shifted from yeast to hyphal growth. Interestingly, both the gdh3/gdh3 mutant and the wild-type strains showed abundant chlamydospores when incubated in arginine medium (Fig. 2). These structures are related to stress and starvation in $C$. albicans. These experiments demonstrated that the gdh3/gdh3 mutant was locked in the yeast morphology when proline was supplied as the sole carbon and nitrogen source.

The growth kinetics of $g d h 2 / g d h 2$ and $g d h 3 / g d h 3$ mutant strains and the parental $C$. albicans strain

To characterise the effect of GDH2 and GDH3 gene deletions on the growth kinetics of $C$. albicans, we monitored the growth of the wild-type and mutant strains on MM, arginine, and proline media at $37^{\circ} \mathrm{C}$ under continuous agitation (200 rpm) (Fig. 3). When the fungal cells were grown on MM medium, there was no significant difference in growth rate $\left(\sim 0.40 \mathrm{~h}^{-1}\right)$ between the wild-type and gdh2/gdh2 strains. The gdh3/gdh3 mutant, however, showed a $24 \%$ reduction in its growth rate $\left(0.302 \mathrm{~h}^{-1}\right)$ compared to wildtype cells.

On the other hand, the gdh3/gdh3 mutant showed similar growth kinetics to the wild-type cells when grown on arginine as the sole carbon and nitrogen source, but the gdh2/gdh2 strain showed minimal growth $\left(0.009 \mathrm{~h}^{-1}\right)$ and biomass yield $\left(0.016 \mathrm{~g} \mathrm{~L}^{-1}\right)$ compared to the wild-type strain (growth rate $=0.068 \mathrm{~h}^{-1}$, biomass $=0.50 \mathrm{~g} \mathrm{~L}^{-1}$ ). Furthermore, the $g d h 2 / g d h 2$ mutant failed to grow on proline as the sole carbon source. This indicated that the gdh2/gdh2 mutation significantly impaired C. albicans utilisation of arginine or proline as the sole carbon and nitrogen source (Fig. 3). The relatively slower growth rate and lower growth yield of the wild-type and ghd3/gdh3 strains on proline or arginine medium, compared to MM medium, can be explained by the fact that amino acids are poor carbon and nitrogen sources in comparison to the glucose and ammonia present in MM medium.

The extracellular metabolite profiles of $g d h 2 / g d h 2$ and $g d h 3 / g d h 3$ mutant strains and the parental C. albicans strain

Thirty-seven metabolites were identified in the spent media of $C$. albicans culture samples (Table 1), and the concentrations of 24 and 19 of these metabolites were significantly different $(p<0.05)$ between wild-type and mutant strains when grown on proline and MM media respectively (Supplementary Fig. 5). In contrast, only 3 extracellular metabolites, 2-isopropylmalate, 4-aminobenzoate, and glutamate, showed significant differences between wild-type and gdh3/gdh3 strains cultured on arginine medium. Interestingly, when growing in proline medium, in which the gdh3/gdh3 mutant cells failed to undergo morphogenesis, they exhibited an overall reduction in extracellular metabolite levels when compared to the wild-type, with the exception of nicotinate, malate, and succinate levels.

The intracellular metabolite profiles of $g d h 2 / g d h 2, g d h 3 / g d h 3$, and parental strains of $C$. albicans

In order to understand how $G D H 2$ and $G D H 3$ gene knockouts influence the metabolic changes associated with $C$. albicans morphogenesis, we compared the intracellular metabolite profiles between wild-type and mutant strains cultured in MM, arginine, or proline media. There were over 100 metabolites detected in the intracellular extracts and 68 of them were identified by our inhouse mass spectral library (Table 1). Among these, the relative concentrations of 62 metabolites were significantly influenced by either $G D H 2$ or $G D H 3$ gene deletions (Fig. 4). Interestingly, when the gdh2/gdh2 mutant utilised glucose as the sole carbon source (MM medium), most of the intracellular metabolites were found at lower concentrations than in the wild-type strain except for cysteine, malonate, nicotinate, 9-heptadecenoate, and 2phosphoenolpyruvate. In contrast, the gdh3/gdh3 mutant showed overall higher concentrations of intracellular metabolites than the wild-type strain when grown in MM medium, except for 2aminobutyrate and norvaline.When grown in a proline medium, the gdh3/gdh3 mutant revealed a significantly higher concentration of glutamate family metabolites (e.g., glutamate, glutamine, and proline) than the wild-type, whilst asparagine, histidine, phenylalanine, valine, leucine, tyrosine, and aspartate family metabolites (e.g., aspartate, isoleucine, and threonine), as well as all saturated fatty acids detected, were at lower concentrations in the gdh3/gdh3 mutant. Coincidentally, valine, leucine, tyrosine, phenylalanine, aspartate, and isoleucine are synthesised by a transamination reaction in which these amino acids gain their amino group by converting glutamate to a-ketoglutarate. When grown in an arginine medium, on the other hand, there were only a few intracellular metabolites for which their concentrations were significantly changed comparing the gdh3/gdh3 and wild-type cells, suggesting that the $g d h 3 / g d h 3$ mutant cells were in a similar metabolic state to the wild-type cells under this environmental condition (Fig. 4). Furthermore, a comparison of the metabolite profiles for gdh3/gdh3 mutants grown in arginine and proline media (Fig. 4, column 5) demonstrated that 20 intracellular metabolites were at higher concentrations in the gdh3/gdh3 mutant cultured in the arginine medium. These metabolites included a range of intermediates from the central carbon 

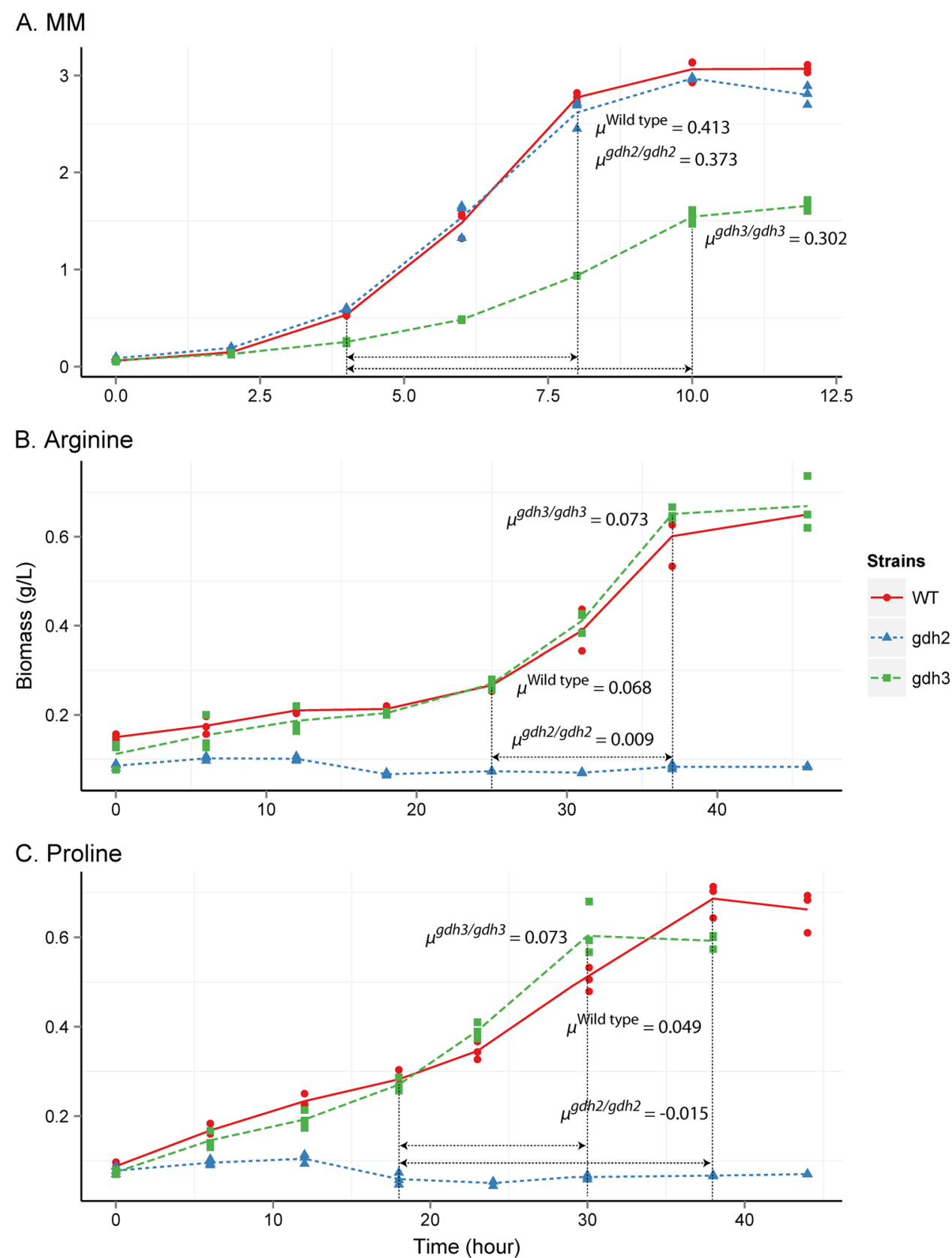

Fig. 3 Growth curves and growth rates for gdh2/gdh2, gdh3/gdh3, and wild-type strains of C. albicans grown on various media. a minimum mineral medium, $\mathbf{b}$ arginine medium, and $\mathbf{c}$ proline medium. All cultures were incubated at $37^{\circ} \mathrm{C}$ with shaking (200 rpm) until the stationary phase was reached. $\mu$ represents the exponential growth rate $\left(h^{-1}\right)$. Five experimental replicates were collected from each growth medium

metabolism including tricarboxylic acid (TCA) cycle intermediates, amino acids, saturated fatty acids, and unsaturated fatty acids. There were only two metabolites, asparagine and $\mathrm{N}$-acetylgluatmate, detected at a lower concentration in the gdh3/gdh 3 cells grown on arginine medium than on proline medium. We investigated how arginine and proline media affected the intracellular metabolites of gdh3/gdh3 and wild-type strains differently (Fig. 4, columns 5 and 6). We detected a lower arginine/proline media ratio (lower metabolite concentrations in arginine medium compared to proline medium) for 2-oxoglutarate, linoleate, 11,14-eicosadienote, proline, 2-aminoadipate, pyroglutamate, para-toluate, 2-aminobutyrate, lysine, glutamine, alanine, glutamate, serine, and margarate in gdh3/gdh3 mutants compared to wild-type. In contrast, we observed a higher arginine/proline media ratio (higher metabolite concentrations in arginine medium compared to proline medium) for succinate, cis-aconitate, citrate, norleucine, $\beta$-alanine, creatinine, citramalate, lysine, aspartate, isoleucine, and 10,13-dimethyltetradecanoate in gdh3/gdh3 mutants compared to wild type (Fig. 4, columns 5 and 6). Changes in these metabolite ratios are likely to be the result of the yeast to hyphae morphological transition.

Effect of $G D H 2$ and $G D H 3$ gene deletions on the metabolic state of C. albicans under hyphae-inducing conditions

The quantification of intracellular metabolites was used to generate a comparative metabolic activity profile for the wildtype and mutant cells grown in MM, arginine, and proline media. Out of the 36 metabolic pathways that showed significant changes in metabolic activity in response to the GDH2 gene 


\begin{tabular}{|c|c|c|c|}
\hline Classification of metabolites & Intra $^{a}$ & Extra $^{\mathrm{b}}$ & Metabolites \\
\hline Amino acid derivatives & 10 & 2 & $\begin{array}{l}\text { Creatinine, cystathionine, } \mathrm{L} \text {-2-aminoadipiate, } \mathrm{N} \text {-acetylglutamate }{ }^{*} \text {, norvaline, ornithine }{ }^{*}, \mathrm{O} \text {-acetyl- } \\
\text { L-serine, } \mathrm{S} \text {-adenosyl-L-homocysteine, and pyroglutamate, } \beta \text {-alanine }\end{array}$ \\
\hline Glycolytic intermediates & 2 & 0 & Pyruvate and phosphoenolpyruvate \\
\hline Cofactors and vitamins & 3 & 1 & NADP/NADPH, nicotinate*, and 4-amino-n-butyrate \\
\hline Antioxidants & 1 & 0 & Glutathione \\
\hline $\begin{array}{l}\text { Total number of identified } \\
\text { metabolites }\end{array}$ & 68 & 37 & \\
\hline
\end{tabular}

deletion when cells were cultured in MM medium, all except phosphonate and phosphinate metabolism were upregulated (Fig. 5). On the other hand, 22 metabolic pathways were significantly downregulated in GDH3-deleted cells cultured in MM medium and only oxidative phosphorylation, sulphur metabolism, biotin metabolism, pyrimidine metabolism, and glycolysis/gluconeogenesis were upregulated. This suggests that the $G D H 2$ and $G D H 3$ genes may play opposite metabolic roles in C. albicans. Interestingly, no metabolic pathways appeared to show significantly different activity between the gdh3/gdh3 mutant and the wild-type strain when grown in arginine medium (Fig. 5, column 4). By comparing the metabolic pathway activities for the gdh3/gdh3 mutant grown on arginine medium as opposed to on proline medium with the wild-type strain under those growth conditions, there was a greater global metabolic reprogramming for the wild-type cells when grown on arginine compared to growth on proline (Fig. 5, columns 5 and 6). Although the wild-type cells retained the same filamentous form in both culture media, by comparing the metabolic activities between arginine and proline media, 16 metabolic pathways were downregulated, whilst 11 metabolic pathways were upregulated for the wild type compared to the gdh3/gdh3 mutant.

${ }^{13} \mathrm{C}$-label distribution through the metabolite profile of $C$. albicans cells grown on $30 \% \mathrm{U}^{13} \mathrm{C}_{6}$ arginine or $30 \% \mathrm{U}-{ }^{13} \mathrm{C}_{5}$ proline as both the sole carbon and nitrogen source, respectively

In order to track how mutants catabolise arginine and proline under hyphae-inducing conditions, we supplied mutant and wildtype strains with $30 \%{ }^{13} \mathrm{C}$-labelled arginine or $30 \%{ }^{13} \mathrm{C}$-labelled proline as the sole carbon source. Our premise was that increased labelling of a metabolite indicates a higher rate of biochemical conversion from the labelled carbon source to the metabolite. Conversely, a decrease in labelling indicates reduced metabolic flux. We found that many metabolites were significantly reduced in one and/or two ${ }^{13} \mathrm{C}$-labelling (see Fig. 6: $M+1$ and $M+2$, which mean one carbon and two carbons labelled, respectively) in the gdh3/gdh3 mutant growing in ${ }^{13} \mathrm{C}$-labelled proline medium, relative to wild-type cells (Fig. 6 , first column). These included TCA cycle intermediates such as citrate, cis-aconitate, a-ketoglutarate, and various amino acids including glutamate, proline, aspartate, asparagine, and alanine as well as glutathione, pyroglutamate, and ornithine. Moreover, we observed an increased $\mathrm{C}^{13}$-labelling of TCA cycle intermediate malate (Fig. 6). Interestingly, some metabolites such as malate, a-ketoglutarate, ornithine, glutamate, ornithine, phenylalanine, tryptophan, tyrosine, leucine, and histidine had higher percentage ${ }^{13} \mathrm{C}$-labelling in three or more carbon atoms $(\mathrm{M}+\geq 3)$ in the gdh3/gdh3 mutant compared to wild-type cells grown in $30 \% \mathrm{U}-{ }^{13} \mathrm{C}_{5}$ proline medium. In contrast, when utilising $30 \% \mathrm{U}^{13}{ }^{13} \mathrm{CR}_{6}$-labelled arginine as the sole carbon source, there were only a few differences between the metabolic labelling in gdh3/gdh3 and wild-type cells that included two TCA cycle intermediates (fumarate and cis-aconitate) and all three aromatic amino acids (phenylalanine, tryptophan, and tyrosine). The difference in aromatic amino acid metabolism may affect the level of quorum sensing molecules, because both phenylalanine, tryptophan, and tyrosine are precursors for the biosynthesis of phenylethyl alcohol, typtophol, and tyrosol respectively. Lastly, in general there were similar labelling patterns when gdh3/gdh3 mutants were compared with the wild-type strain grown on proline as when gdh3/gdh3 cells grown in proline and in arginine media were compared (column 1 and column 3 in Fig. 6).

\section{Cofactor concentrations in C. albicans wild-type and mutant} strains

The concentrations of ATP, NAD ${ }^{+}, \mathrm{NADH}, \mathrm{NADP}^{+}$, and NADPH in $\mathrm{C}$. albicans cells were measured in order to determine the energetic and redox state of the different strains under different growth conditions (Figs 7 and 8). The oxidised forms of $\mathrm{NAD}^{+}$and NADP ${ }^{+}$ were found at higher concentrations than the reduced forms in samples from all C. albicans strains under the different growth conditions (Fig. 8). The gdh3/gdh3 mutant incubated in arginine medium exhibited significantly lower $\mathrm{NAD}^{+}, \mathrm{NADH}, \mathrm{NADP}^{+}$, and NADPH concentrations $(p<0.001)$ compared to the wild-type cells (Fig. 8), while ATP remained at a similar concentration (Fig. 7). In contrast, the gdh3/gdh3 mutant showed a higher concentration of ATP, NAD ${ }^{+}$, and $\mathrm{NADH}$, but not $\mathrm{NADP}^{+}$or $\mathrm{NADPH}$, when incubated in proline medium when compared to the wild-type 


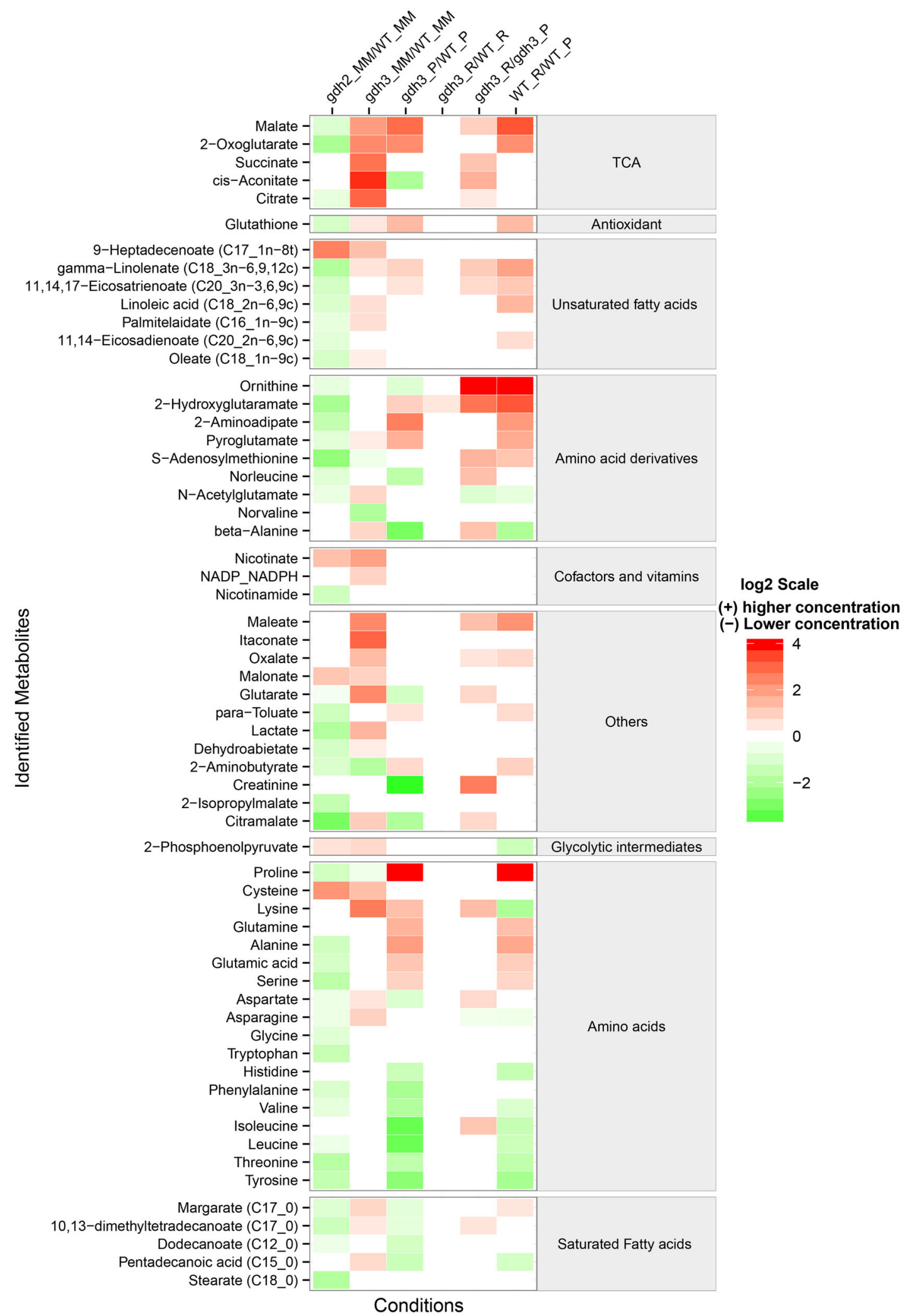

Fig. 4 The ratio of intracellular metabolite concentrations between $C$. albicans strains when cultured on arginine (R), proline (P), or minimum mineral (MM) media. The metabolite concentrations relative to those in the wild-type (except column 5 which is relative to gdh3 cells grown in proline medium) have been plotted using a $\log _{2}$ scale. The red colour (positive values) indicates that metabolite concentrations were higher in numerator strains than the denominator (comparator) strains, while green shades (negative values) indicate reduced concentrations in numerator strains compared to the denominator strains. Only the metabolites for which there was a statistically significant change in concentration between the wild-type and mutant strains (Tukey's honest significance test, $p$-value $<0.05$ ) are shown 


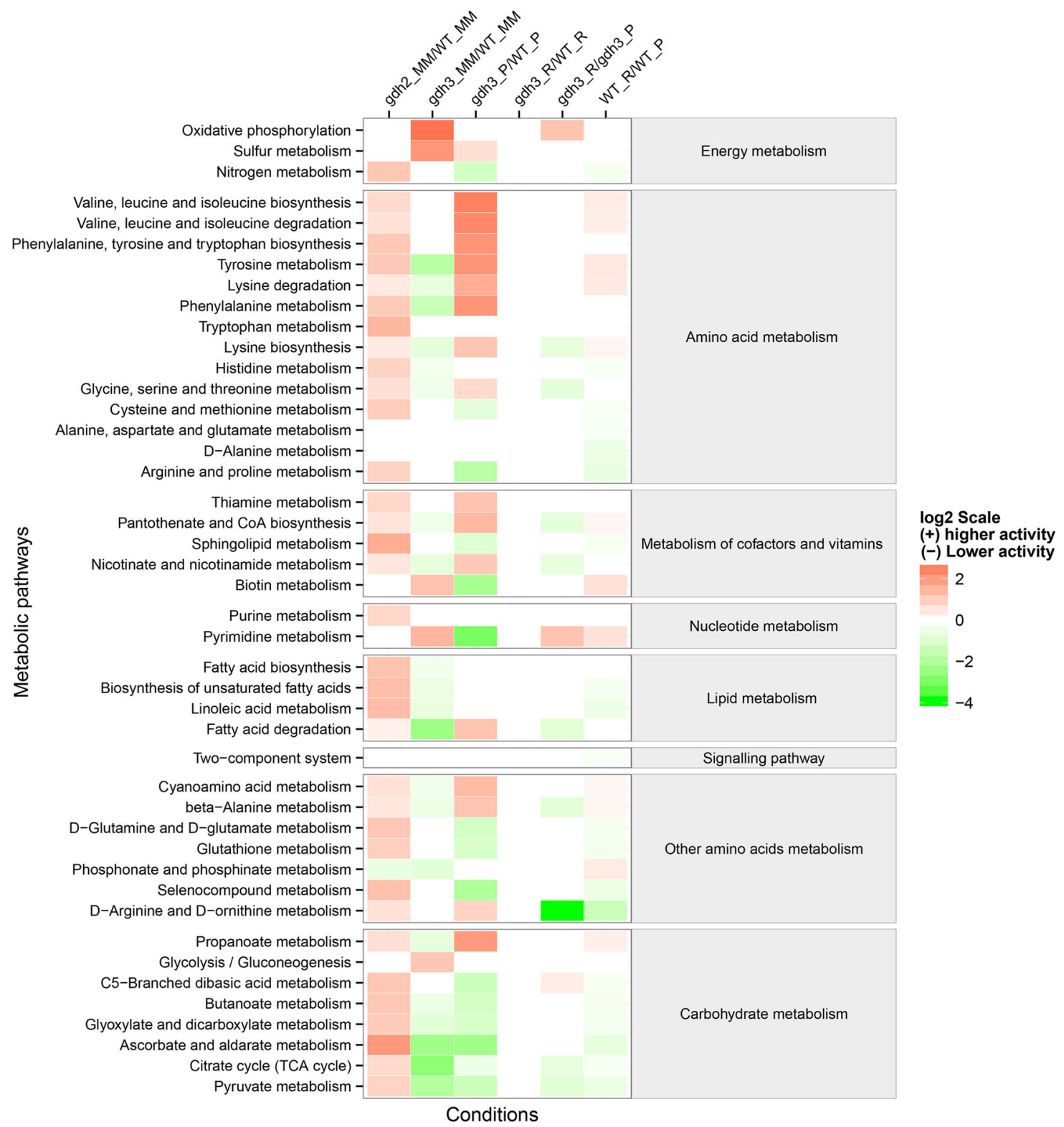

Fig. 5 Activities of $C$. albicans metabolic pathways predicted from intracellular metabolomics data from the wild-type (WT) and gdh2/gdh2 or gdh3/gdh3 strains grown in various culture media. MM is minimum mineral medium, $\mathrm{P}$ is proline medium, and $\mathrm{R}$ is arginine medium. The metabolic pathway activities relative to the denominator strains are shown using a $\log _{2}$ scale. Red colours (positive values) mean pathways that were upregulated in numerator strain compared to the denominator (comparator) strain. Green colours (negative values) indicate pathways that had their activity downregulated in numerator strain compared to the denominator strain. Only the metabolic pathways for which there was a statistically significant change in activity (Tukey's honest significance test, $p$-value $<0.05$ ) are shown

strain (Figs 7 and 8). The higher concentrations of ATP, NAD ${ }^{+}$, and $\mathrm{NADH}$ could explain why the gdh3/gdh3 mutant remained in the yeast form in arginine medium (hyphae-inducing conditions). In MM medium, only the gdh3/gdh3 mutant showed a significantly lower concentration of $\mathrm{NADH}, \mathrm{NADP}^{+}$, and NADPH than the wildtype strain. Furthermore, when the ratios of $\mathrm{NAD}^{+}$to $\mathrm{NADH}$ in wild-type and mutant strains were compared we detected a significantly lower ratio of $\mathrm{NAD}^{+}$to $\mathrm{NADH}$ in the gdh3/gdh3 mutant than in the wild-type strain when incubated in proline medium, while a higher ratio of $\mathrm{NAD}^{+}$to $\mathrm{NADH}$ in the gdh3/gdh3 mutant than in the wild-type strain in MM medium (Supplementary Fig. 6). The decreased ratio of $\mathrm{NAD}^{+}$to $\mathrm{NADH}$ in the gdh3/ gdh3 mutant indicates that cofactor equilibrium may be important for the morphogenesis of $C$. albicans.

\section{DISCUSSION}

This study implicates $G D H 2$ and $G D H 3$ gene knockout mutagenesis, metabolomics, and isotope labelling approaches to elucidate how the fungus $C$. albicans changes its cellular metabolism during the yeast-to-hyphal transition. There were three major comparisons performed in this study in order to determine the role of $G D H 2$ and $G D H 3$ in C. albicans growth and morphogenesis. Firstly all three strains, the $G D H 2$ and $G D H 3$ deletion mutants and the wild-type strain, grown in MM medium were compared to investigate the metabolic differences between mutant and wildtype strains during the morphological transition (gdh2-MM vs WTMM, gdh3-MM vs WT-MM). Secondly we compared the metabolic profiles of the gdh3/gdh3 mutant and wild type when grown in arginine and proline media (gdh3-P vs WT-P, gdh3-R vs WT-R). Since the $g d h 2 / g d h 2$ mutant was unable to grow, when arginine 


\section{TCA cycle intermediates}

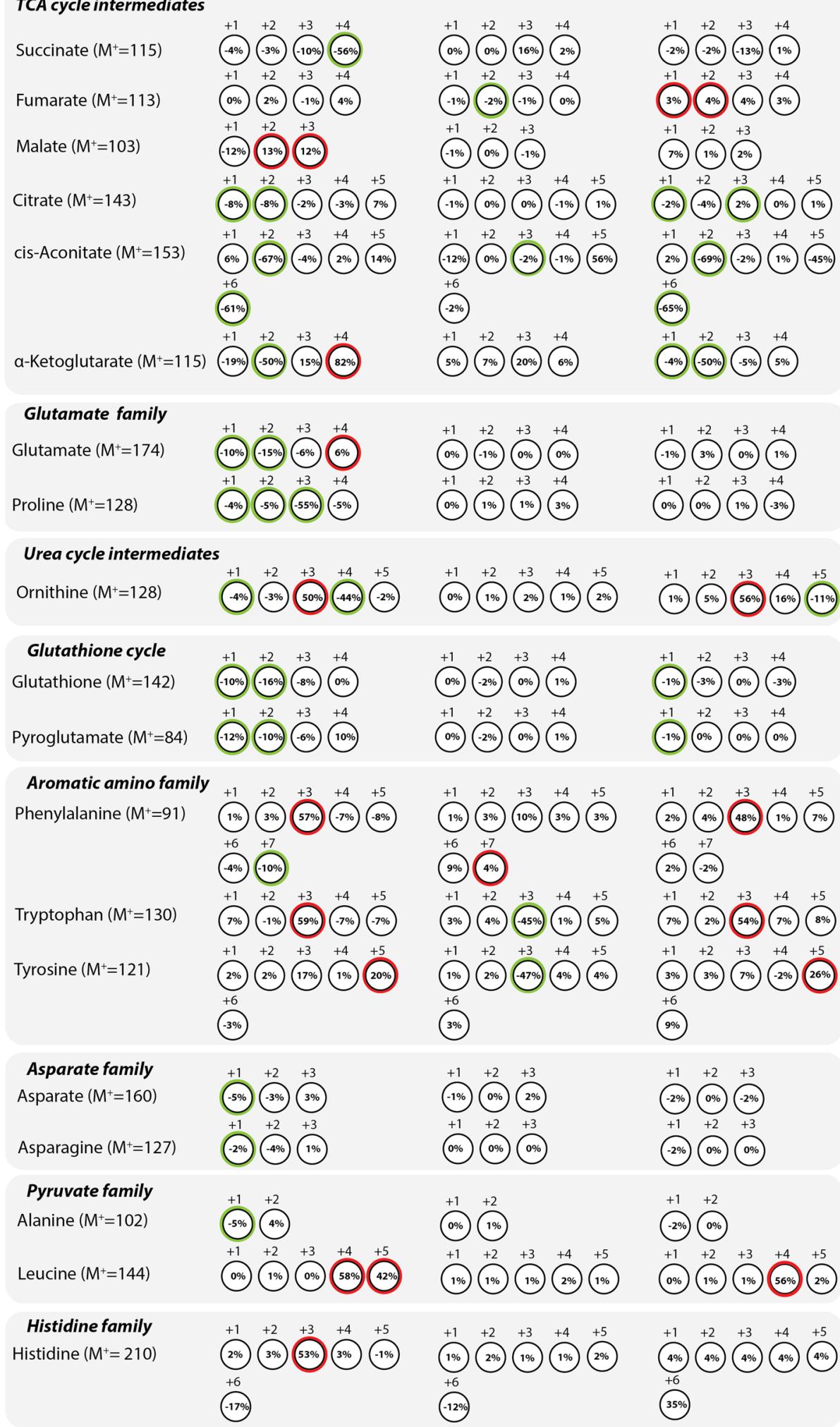

Fig. 6 The incorporation of ${ }^{13} \mathrm{C}$ carbon atoms from $\mathrm{U}^{13} \mathrm{C}_{5}$ proline or $\mathrm{U}-{ }^{13} \mathrm{C}_{6}$ arginine into metabolites from the wild-type and mutant strains of C. albicans. The figure indicates the percentage difference of ${ }^{13} \mathrm{C}$-labelling between two cell types when utilising $30 \% \mathrm{U}-{ }^{13} \mathrm{C}_{6}$ arginine or $30 \%$ $\mathrm{U}_{-}^{13} \mathrm{C}_{5}$ proline as both the sole carbon and nitrogen source, respectively. $\mathrm{M}^{+}$is the main molecular ion of an identified metabolite. +1 is $1 \mathrm{~m} / \mathrm{z}$ higher than the $\mathrm{M}^{+}$. Only the metabolite ions for which there was a statistically significant change in the percentage of ${ }^{13} \mathrm{C}$-atom between the cell types ( $p$-value $<0.05$ and $>2$-fold differences) are highlighted with coloured circles. Green circles represent lower isotope labelling in the first strain. Red circles indicate higher isotope labelling in the first strain 


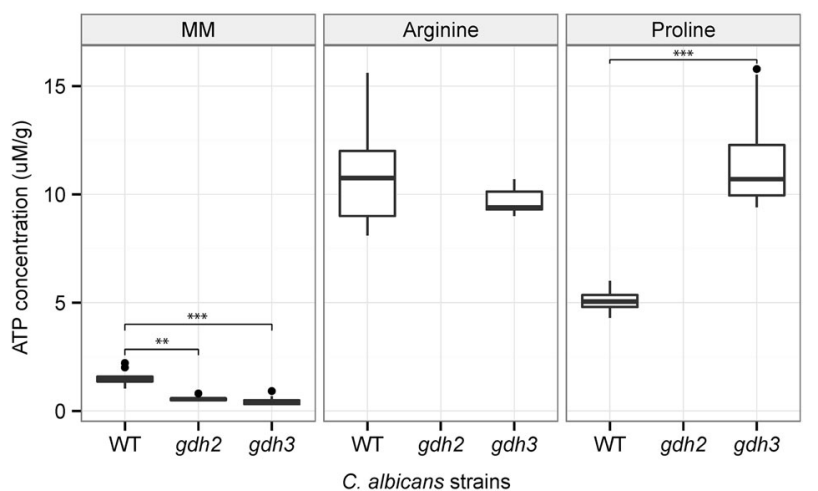

Fig. 7 ATP concentrations in the wild-type (WT), gdh2/gdh2, and gdh3/ gdh3 strains of C. albicans incubated in the three different media. The ATP pools in mutant cells grown in (MM) minimum mineral and proline media were significantly different from the wild-type but not cells cultured in arginine medium. ATP was not measured in the gdh2/ gdh2 mutant incubated in arginine or proline media because this mutant was unable to grow under these conditions. The unit for ATP concentration is $\mu$ mole per gram of cell biomass. Fifteen experimental replicates were performed for each group. The distributions of boxplot are minimum, 25th percentile, median, 75th percentile, and maximum (from bottom to upper direction). Black dots are outliers ( $>1.5$ times of interquartile range). ${ }^{* *}$ Tukey's honest significance test $(p$-value $<0.05)$ and ${ }^{* * *}(p$-value $<0.001)$

and proline were provided as the sole carbon and nitrogen sources, this strain could not be used in this experiment. The last comparison was of the concentrations of different metabolites between arginine and proline media for gdh3/gdh3 mutant compared to those in the wild-type strain under the same conditions (comparison between gdh3-R/gdh3-P and WT-R/WT-P). The purpose of the last comparison was to determine the metabolic differences in the gdh3/gdh3 mutant caused by different growth morphologies. We could do this because the gdh3/gdh3 mutant was unable to undergo morphogenesis in the proline medium but became highly filamentous in the arginine medium (Fig. 2). This enabled us to remove the metabolic effect from the use of different media.

We expected that the gdh2/gdh2 and gdh3/gdh3 mutations would induce a significant alteration in the redox potential of the $C$. albicans cells when we deleted these genes, as previously observed in S. cerevisiae ${ }^{9}$, and hypothesised that this would also affect morphogenesis. Indeed, we found that the concentrations of oxidised and reduced forms of NAD and NAPD were significantly changed in the gdh2/gdh2 and ghd3/gdh3 mutants compared to the wild-type strain in $\mathrm{MM}$, arginine and proline media. The deletion of the GDH2 or GDH3 gene in C. albicans, however, did not seem to affect its morphogenetic ability greatly in MM medium. This implied that the redox potential of the cell alone may not be enough to affect the morphogenesis of $C$. albicans. On the other hand, the yeast form of the gdh3/gdh3 mutant showed a significantly higher intracellular concentration of ATP, NAD ${ }^{+}, \mathrm{NADH}, \mathrm{NADP}^{+}$, and NADPH compared to the filamentous form of the wild-type strain in proline medium. These results supported the hypothesis we previously proposed ${ }^{8}$ that once the metabolic activity of yeast cells is temporarily disrupted by environmental stresses, the central carbon metabolism of $C$. albicans is globally downregulated. It is proposed that this downregulation of the central carbon metabolism triggers changes to the growth form as a result of less energy being available, and consequently impacts filamentous growth. We speculated that hyphae exhibit lower metabolic activities due to the necessity of utilising alternative carbon sources in contrast to the dominance of sugar metabolism in the yeast form. C. albicans often lives in the gastrointestinal tract of its mammalian host and on epithelial surfaces, where there are diverse and can be abundant carbon sources. Whilst competition with other microorganisms for nutrients is intense, an ability to assimilate alternative carbon and nitrogen sources, and to enable directional growth with filament development, is no doubt an advantageous survival strategy. Indeed, the gdh3/gdh3 mutant showed reduced assimilation of proline as a carbon and nitrogen source, and with increased ATP concentrations, this may be the reason that the gdh3/gdh3 mutant stayed in the yeast form.

Although the gdh3/gdh3 mutant demonstrated similar growth behaviour as the wild-type strain in proline and arginine media, it showed attenuated growth in MM medium. We suggest that this is because the $g d h 3 / g d h 3$ mutant is unable to efficiently assimilate nitrogen sources into the central carbon metabolism. Gdh3 favours the assimilation of ammonium through the anabolic reaction with $a$-ketoglutarate to form glutamate. Since ammonium is the only nitrogen source in MM medium, the $g d h 3 / g d h 3$ mutant may rely on Gdh2 to incorporate ammonium into glutamate. This process will consume NADH and compromise the use of NADH for the production of ATP via the electron transport chain. Indeed, we observed a lower ratio of NADH to $\mathrm{NAD}^{+}$(Supplementary Fig. 6A) and a lower level of ATP (Fig. 7) in the gdh3/gdh3 mutant when it was grown in MM medium. The metabolic pathway analysis (Fig. 5) also predicted a global downregulation of central carbon metabolism in the $g d h 3 / g d h 3$ mutant compared to the wild-type cells in MM medium. On the other hand, the ghd3/gdh3 mutation did not reduce growth rate in either the arginine or proline medium compared to the wildtype (Fig. 3). We propose this is because Gdh2 is still present and has a high affinity for the enzymatic deamination of glutamate into a-ketoglutarate. Therefore, it is not surprising that the $G D H 3$ deletion did not influence growth significantly because it is substituted by Gdh2.

The gdh2/gdh2 mutant failed to utilise arginine and proline as both the sole carbon and nitrogen sources. Indeed, the biomass of the $g d h 2 / g d h 2$ mutant gradually reduced over $40 \mathrm{~h}$ of incubation in the proline and arginine media (Fig. 3). Both arginine and proline belong to the glutamate family of amino acids. When these amino acids are used as carbon and nitrogen sources, they must be converted into glutamate, followed by a transamination reaction into a-ketoglutarate and ammonia, which is then catabolised by glutamate dehydrogenase. Therefore, the deletion of the GDH2 gene will certainly impact on the catabolism of arginine and proline, considering that Gdh2 is the main enzyme capable of converting glutamate into a-ketoglutarate through the reduction of $\mathrm{NAD}^{+}$. Furthermore, the inability to utilise arginine and proline could also suggest that both PUT and GLN1/GLT1 pathways (Fig. 1) are dysregulated in response to the $G D H 2$ gene deletion, or these pathways are unable to generate sufficient glutamate inside the cells and the large pool of free $\mathrm{NADP}^{+}$ required to overcome the thermodynamic barrier inherent in using the still present Gdh3, which is usually an anabolic enzyme with a higher affinity for a-ketoglutarate than for glutamate. The lack of central carbon and nitrogen catabolism not only leads to energy deficiency but also reduces the supply of the main metabolite anabolic precursors such as pyruvate, acetyl-CoA, glucose-6-phosphate, and erythrose 4-phosphate used for biomass biosynthesis ${ }^{21}$. These effects are clearly demonstrated by growth attenuation (Fig. 3) and the lack of hyphal formation (Fig. 2) in the gdh2/gdh2 mutant when proline or arginine were supplied as the sole carbon and nitrogen sources. In contrast, the gdh2/gdh2 mutant can grow in MM medium because aketoglutarate is supplied from glucose via glycolysis.

We postulate that $\mathrm{NAD}^{+} / \mathrm{NADH}$ disequilibrium in the gdh $3 / g d h 3$ mutant led to a different proline catabolism in the mutant compared to the wild-type strain. Our isotope labelling results (Fig. 6) demonstrated that the gdh3/gdh3 mutant exhibited a lower percentage of ${ }^{13} \mathrm{C}$ labelling of one to two carbon atoms in 

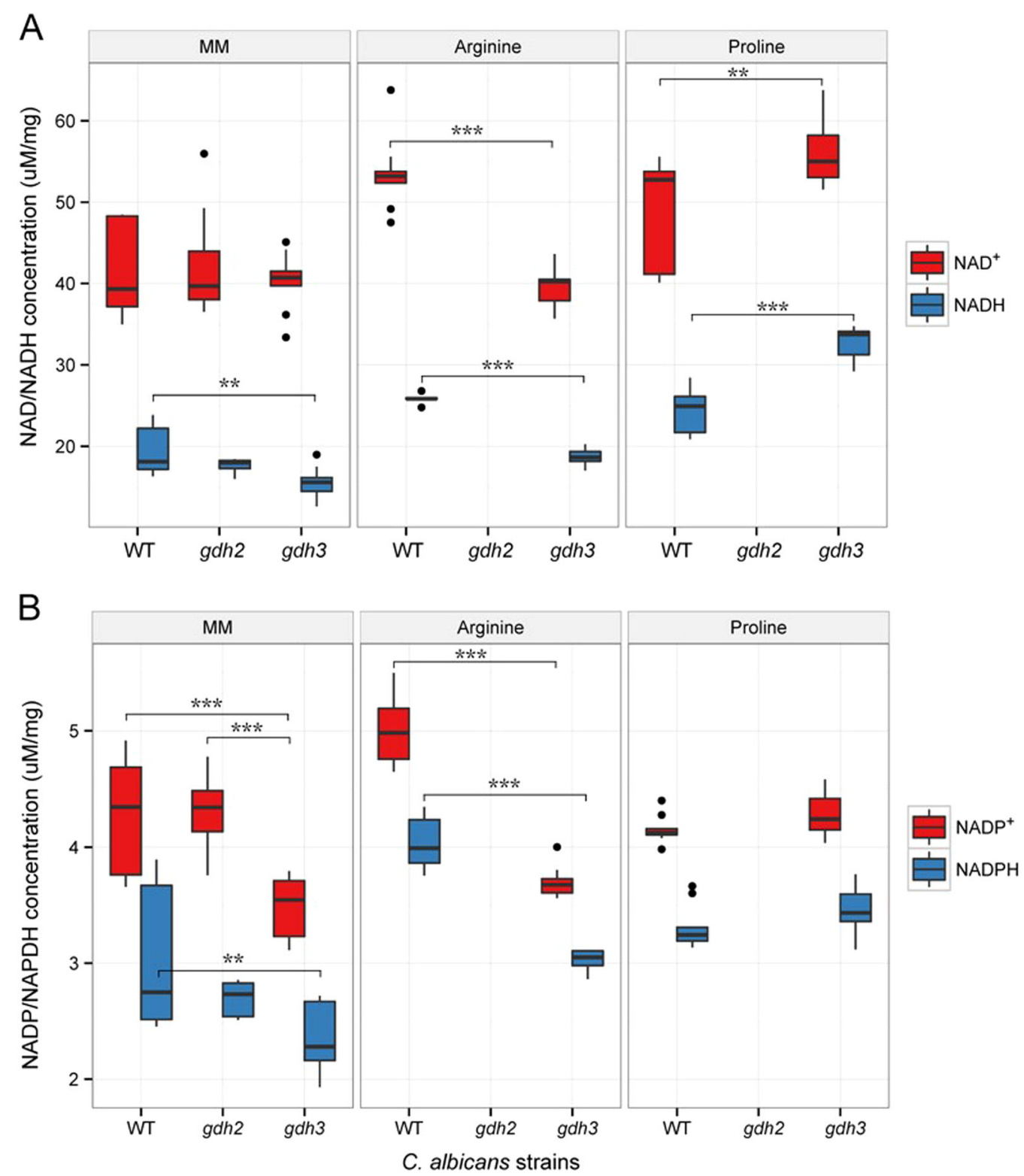

Fig. 8 Cofactor concentrations in the wild-type (WT), gdh2/gdh2, and gdh3/gdh3 strains of C. albicans incubated in arginine, proline, or minimum mineral (MM) media. a The concentration of $\mathrm{NAD}^{+}$(red boxes) and $\mathrm{NADH}$ (blue boxes) found in C. albicans mutant and wild-type strains. b The concentration of $\mathrm{NADP}^{+}$(red boxes) and NADPH (blue boxes) detected in C. albicans mutant and wild-type strains. Cofactor concentrations were not measured in the gdh2/gdh2 mutant under arginine and proline media because this mutant was unable to grow under these conditions. The unit for NADP/NADPH concentration is $\mu$ mole per gram of cell biomass. Nine experimental replicates were collected and measured from each group. The distributions of boxplot are minimum, 25th percentile, median, 75 th percentile, and maximum (from bottom to upper direction). Black dots are outliers ( $>1.5$ times of interquartile range). Tukey's honest significance test ${ }^{* *}(p$-value $<0.05)$ and ${ }^{* * *}(p$-value $<0.001)$

metabolites while a higher percentage of ${ }^{13} \mathrm{C}$ labelling for three or more carbon atoms in metabolites for most of TCA cycle intermediates and amino acids when compared to the wildtype. This result could be explained by the fact that catabolism of proline in the gdh3/gdh3 mutant involves only $\mathrm{NAD}^{+}$-dependent reactions, including the Gdh2- and Put2-catalysed reactions. The PUT2 gene encodes pyrroline 5-carboxylate dehydrogenase which catalyses the conversion of pyrroline-5-carboxylate and $\mathrm{NAD}^{+}$to glutamate (Fig. 1). This reaction is crucial for proline degradation when used as the sole source of carbon and nitrogen. Therefore, when proline is the only carbon and nitrogen source, the gdh3/ gdh3 cells would probably be experiencing a serious shortage of the $\mathrm{NAD}^{+}$required to catabolise proline via the PUT pathway. We found an accumulation of proline, glutamate, 2-oxoglutarate, malate, and a lower $\mathrm{NAD}^{+}$to $\mathrm{NADH}$ ratio inside the gdh3/gdh3 mutant relative to wild-type cells when grown in proline medium. This indicated a lower influx of proline into the TCA cycle via the glutamate to a-ketoglutarate route. On the other hand, the wildtype strain could relieve the high $\mathrm{NAD}^{+}$usage by employing Gdh3 which is dependent on $\mathrm{NADP}^{+}$. There are also alternative proline catabolic pathways that cleave proline into two-carbon molecules, yet are $\mathrm{NAD}^{+}$dependent, so these metabolic pathways are suppressed in the gdh3/gdh3 mutant. Since preferred nitrogen sources, such as ammonia and nitrogen were not present when 
proline was used as the sole carbon and nitrogen source, catabolite repression of the proline utilisation pathway (PUT1 and PUT2) would not have occurred ${ }^{19}$. Surprisingly, a higher concentration of ATP was detected in the gdh3/gdh3 mutant than in the wild-type strain. This could result from the higher NADH production by Gdh2 as well as an upregulation of $\beta$-oxidation. In proline medium, we observed a lower concentration of saturated fatty acids (Fig. 4) and higher metabolic activity involved in fatty acid degradation (Fig. 5) in the gdh3/gdh3 mutant than in the wild-type strain. Since $\beta$-oxidation catabolises fatty acids into $\mathrm{NADH}, \mathrm{FADH}_{2}$, and acetyl-CoA, which enters the TCA cycle, upregulated $\beta$-oxidation results in the degradation of fatty acids and increased ATP production.

Why is hyphal formation in the gdh3/gdh3 mutant only suppressed in proline medium but not in arginine medium when both amino acids belong to the glutamate amino acid family? We also observed no significant differences in the metabolic pathway activity (Fig. 5) or cofactor ratio (Supplementary Fig. 6A) between the gdh3/gdh3 mutant and wild-type strain when grown in arginine medium. The in silico model of the C. albicans central carbon metabolism proposed by $\mathrm{Han}$ et al. ${ }^{10}$ proposed that arginine could bypass the bottleneck of glutamate dehydrogenase and enter the TCA cycle via the urea cycle. Arginine is part of the urea cycle and can be rapidly cleaved to urea and ornithine by the arginase enzyme (Car1). The ornithine is condensed by Arg3 with carbamyl phosphate and forms citrulline. This is then condensed by Arg1 with aspartate to form argininosuccinate which is then cleaved to fumarate-one of the TCA cycle intermediates. We also predict that urea, cleaved from arginine, can be hydrolysed into two carbon dioxide molecules and ammonia. This ammonia can feed into the GLN1/GLT1 pathway and be converted into glutamate at the expense of ATP and NADH (Fig. 1). These pathways produce $\mathrm{NAD}^{+}$which can be used to convert glutamate to a-ketoglutarate via $\mathrm{NAD}^{+}$-dependent $G D H 2$. Indeed, we observed a similar $\mathrm{NAD}^{+}$and $\mathrm{NADH}$ ratio between the ghd3/ gdh3 mutant and the wild-type when grown in arginine medium (Supplementary Fig. 6A). Lastly, additional genetic manipulations should be performed such as restoring the deleted GDH genes or overexpression $G D H$ genes in the knockout mutants. If the phenotypes of the mutants can be successfully restored to wild type, this will confirm that only the correct genes have been deleted and further validate the specific phenotypes ascribed to each $G D H$ mutant.

Lastly, the results of this study consistently indicated the importance of redox potential and nitrogen metabolism to the regulation of hyphal development. We observed differential morphogenetic responses between the gdh3/gdh3 mutant and the wild-type strain when grown on proline as the sole carbon and nitrogen source. We propose that $\mathrm{NADH}$-dependent glutamate dehydrogenase activity is favoured over NADPH production to meet the immediate energy requirements of the starved wild-type cells. The excess production of NADH during the catabolism of proline in the gdh3/gdh3 mutant could be at the expense of NAD ${ }^{+}$ supply and formation of the NADPH needed for cellular division and hyphal development. On the other hand, arginine overcomes the disequilibrium of the cells' redox balance through the bypass of glutamate dehydrogenase and enters the TCA cycle via the urea cycle. Further studies such as generating knockout mutations in other genes involved in maintaining the cell's redox balance could also be undertaken to better characterise $C$. albicans morphogenesis in relation to central carbon metabolism. These studies could identify key enzymes involved in maintaining the cell's redox balance. Such enzymes could be developed as targets which when inhibited would block the morphological transition in $C$. albicans-a major virulence factor for this opportunistic pathogen -and provide additional therapeutic options to overcome $C$. albicans infections.

\section{METHODS}

Candida albicans strains and plasmids

C. albicans strain $\mathrm{SC} 5314^{22}$ was used as the wild-type parental strain from which mutants were generated (Table 2). The SAT1 flipper fragment containing the nourseothricin resistance gene was amplified from plasmid pSFS2 ${ }^{20}$. Ethics approval to develop genetically modified C. albicans strain SC5314 was granted by the Environment Risk Management Authority of New Zealand (ER-AF-NO3P-3 14/11).

\section{Chemicals and reagents}

All chemicals used in this study were of analytical grade. Methanol, chloroform, sodium bicarbonate, and sodium hydroxide were obtained from MERK (Damstadt, Germany). The internal standard 2,3,3,3- $\mathrm{d}_{4}$-alanine, the derivatisation reagent methyl chloroformate (MCF), and pyridine were purchased from Sigma-Aldrich (St. Louis, USA). Anhydrous sodium sulphate and farnesol were obtained from Fluka (Steinheim, Germany). $\mathrm{U}-{ }^{13} \mathrm{C} 5$ L-proline and $\mathrm{U}^{13} \mathrm{C} 6$ arginine were acquired from Cambridge Isotope Laboratory Inc. (Tewksbury, USA). Two types of DNA polymerases were utilised in this study: KOD hot start DNA polymerase from NovgenR (Darmstadt, Germany), used to construct the SAT1 flipper disruption cassette, and TaKaRa Ex TaqTM from Takara Bio Inc. (Shiga, Japan), used for colony PCR amplifications. The antibiotic nourseothricin was purchased from WERNER BioAgents (Jena, Germany). The QIAquick Gel Extraction kit was supplied by Qiagen (Valencia, CA, USA) and the Alkali-cation yeast transformation kit was obtained from Qbiogene (Heidelberg, Germany).

\section{Primers designed for this study}

Primers were designed using Oligo 6 software (MBI, USA) and synthesised by either Invitrogen ${ }^{\mathrm{TM}}$ (Victoria, Australia) or IDT (Leuven, Belgium). A list of DNA primers used in this study is presented in Supplementary Table 1.

\section{Construction of SAT1 flipper disruption cassette}

Both alleles of target genes were deleted from C. albicans SC5314 using the $S A T 1$ flipper method ${ }^{20}$. Briefly, $\sim 0.4 \mathrm{~kb}$ DNA upstream ( $5^{\prime}$ flank) of the open reading frame (ORF) was amplified by PCR of genomic DNA using primers P1 and P2 (Supplementary Table 1 and Supplementary Fig. 1). Similarly, $\sim 0.4 \mathrm{~kb}$ DNA downstream ( $3^{\prime}$ flank) of the open reading frame (ORF) was amplified by PCR of genomic DNA using primers P3 and P4. The SAT1 flipper fragment was amplified from plasmid pSFS2 ${ }^{20}$ using primers $\mathrm{P} 5$ and P6 that contained $5^{\prime}$ extensions, which were complementary to the $5^{\prime}$ ends of primers $\mathrm{P} 2$ and $\mathrm{P} 3$, respectively. Lastly, all three purified DNA fragments were joined together by fusion $P C R^{23}$ using primers $\mathrm{P} 1$ and $\mathrm{P} 4$ to complete the construction of SAT1 flipper disruption cassette. The SAT1 flipper disruption cassette was isolated and gel-purified using the QIAquick Gel Extraction kit (Qbiogene). In brief, the band containing the DNA cassette was excised from the agarose gel and incubated with binding buffer at $50^{\circ} \mathrm{C}$ for $10 \mathrm{~min}$. After complete dissolution of the gel, the sample was added to a Qiagen spin column which was then centrifuged at $9200 \times g$ for $1 \mathrm{~min}$. The spin column was washed with $0.75 \mathrm{~mL}$ of washing buffer followed by centrifugation at $9200 \times g$ for $1 \mathrm{~min}$, twice. The DNA cassette was eluted from the spin column by adding $50 \mu \mathrm{L}$ of elution buffer and the column was centrifuged at $15,700 \times g$ for $1 \mathrm{~min}$.

Table 2. C. albicans wild-type and mutant strains used in this study

\begin{tabular}{|c|c|c|}
\hline Strains & Genotypes & References \\
\hline SC5314 & Wild-type & 22 \\
\hline GDH2_M1 & SC5314:: GDH2/gdh2 $2::: S A T 1-F L P-F R T^{a}$ & This study \\
\hline GDH2_M2 & $\mathrm{SC} 5314:: \mathrm{GDH} 2 / \mathrm{gdh} \Delta::: \mathrm{FRT}^{\mathrm{b}}$ & This study \\
\hline GDH2_M3 & SC5314:: gdh2 $\Delta::: F R T / g d h 2 \Delta::: S A T 1-F L P-F R T$ & This study \\
\hline GDH2_M4 & SC5314:: gdh2 $2:::$ FRT/gdh2 $2 \Delta:: F R T$ & This study \\
\hline GDH3_M1 & SC5314:: GDH3/gdh3 $\Delta::$ SAT1-FLP-FRT & This study \\
\hline GDH3_M2 & SC5314:: GDH3/gdh3 $4:: F R T$ & This study \\
\hline GDH3_M3 & SC5314:: gdh3 $\Delta:: F R T / g d h 3 \Delta:: S A T 1-F L P-F R T$ & This study \\
\hline GDH3_M4 & SC5314:: gdh3 $3:: F R T / g d h 3 \Delta:: F R T$ & This study \\
\hline
\end{tabular}

aSAT1-FLP-FRT denotes SAT1 flipper cassette

${ }^{\mathrm{b}} \mathrm{FRT}$ is the abbreviation for FLP recombination target 


\section{C. albicans transformation and selection of transformants}

C. albicans strain SC5314 was transformed by a combination of heat shock and lithium/caesium acetate provided by the Alkali-cation yeast transformation kit (Qbiogene). The transformation protocol was adapted from the kit instructions and the method described by Hernday et al. ${ }^{24}$. A colony of C. albicans was used to inoculate $10 \mathrm{~mL}$ of YPD medium $\left(6 \mathrm{gL}^{-1}\right.$ yeast extract, $3 \mathrm{gL}^{-1}$ peptone, and $10 \mathrm{gL}^{-1}$ glucose at $\mathrm{pH}$ 6.5) in shake flasks which was incubated overnight at $30^{\circ} \mathrm{C}$ in a rotary shaker. This culture was then diluted into $30 \mathrm{~mL}$ of YPD medium to give an initial $\mathrm{OD}_{600}$ of 1.0 and incubated at $30^{\circ} \mathrm{C}$ in the rotary shaker for $4 \mathrm{~h}$. After centrifugation at $15,700 \times g$ for 5 min the supernatant was discarded and the cell pellet was repeatedly washed in $5 \mathrm{~mL}$ of TE buffer and resuspended in $3 \mathrm{~mL}$ of lithium/caesium acetate solution (Qbiogene). The cell suspension was incubated for a further $30 \mathrm{~min}$ at $30^{\circ} \mathrm{C}$ with shaking. The cells were then isolated by centrifugation and resuspended in $300 \mu \mathrm{L}$ of TE buffer. The following solutions were mixed together: $100 \mu \mathrm{L}$ of cells in TE buffer, $10 \mu \mathrm{L}$ of herring sperm DNA $\left(10 \mathrm{mg} \mathrm{mL}^{-1}\right), 10 \mu \mathrm{L}$ of SAT1 flipper disruption cassette $\left(0.1 \mu \mathrm{gL}^{-1}\right)$, and $5 \mu \mathrm{L}$ of histamine solution (Qbiogene). The mixture was incubated at room temperature for $15 \mathrm{~min}$, followed by adding $1 \mathrm{~mL}$ of diluted PEG $(0.8 \mathrm{~mL}$ of PEG $+0.2 \mathrm{~mL}$ of TE/Cation - Kit components) and incubated at room temperature for another $3 \mathrm{~h}$. After this, the cells were exposed to heat shock at $42^{\circ} \mathrm{C}$ for $1 \mathrm{~h}$. The cells were isolated by centrifugation and allowed to recover in $5 \mathrm{~mL}$ of YPD broth at $30^{\circ} \mathrm{C}$ in a rotary shaker for $5 \mathrm{~h}$ prior to selective growth. Yeast cells were spread on YPD agar plates (YPD medium with $15 \mathrm{gL}^{-1}$ agar) containing nourseothricin $\left(200 \mathrm{\mu g} \mathrm{mL}^{-1}\right)$ and incubated at $30^{\circ} \mathrm{C}$ for 3 days. Nourseothricin-resistant colonies that contained correct $5^{\prime}$ and $3^{\prime}$ junctions in the disrupted alleles were confirmed by colony PCR using primers $\mathrm{C} 1$, C2, C3, and C4 (Supplementary Fig. 1 and Table 2).

The excision of the SAT1 flipper cassette was induced by inoculating $5 \mathrm{~mL}$ of YPD broth, containing $2 \%$ maltose (without nourseothricin), with the nourseothricin-resistant strains and incubating them in a rotary shaker at $30^{\circ} \mathrm{C}$ overnight. Approximately 100 cells were plated on YPD agar containing nourseothricin $\left(25 \mu \mathrm{g} \mathrm{mL}^{-1}\right)$ and incubated at $30^{\circ} \mathrm{C}$ for 2 days. Slow growing colonies were patched onto YPD agar plates with and without nourseothricin $\left(200 \mu \mathrm{g} \mathrm{mL}^{-1}\right)$ to screen for nourseothricinsensitive colonies. The excision of the SAT1 flipper cassette was also confirmed by colony PCR using primers $\mathrm{C} 1$ and $\mathrm{C} 4$. The entire transformation procedure was repeated to delete both copies of $G D H$ alleles. Lastly, a final PCR verification using primers $\mathrm{C} 5$ and $\mathrm{C6}$ (Supplementary Fig. 1 and Table 2) was performed to confirm that no copies of the $G D H$ alleles or the SAT1 flipper disruption cassette were remaining in C. albicans gdh/gdh homozygous mutants.

\section{Morphology of mutant and wild-type strains}

C. albicans strains were maintained on YPD agar plates at $30^{\circ} \mathrm{C}$. These cells were used to inoculate $100 \mathrm{~mL}$ of YPD broth which was incubated at $30^{\circ} \mathrm{C}$ in a rotary shaker overnight. The cells were collected by centrifugation at $2000 \times g$ for $5 \mathrm{~min}$ and washed in phosphate buffered saline $\left(8 \mathrm{gL}^{-1} \mathrm{NaCl}\right.$, $0.2 \mathrm{gL}^{-1} \mathrm{KCl}, 1.44 \mathrm{gL}^{-1} \mathrm{Na}_{2} \mathrm{PO}_{4}, 0.24 \mathrm{gL}^{-1} \mathrm{KH}_{2} \mathrm{PO}_{4}$, at $\mathrm{pH}$ 7.5). The cells were resuspended in the desired growth media to an initial optical density $\left(\mathrm{OD}_{600}\right)$ of 0.1 . To study the morphology of wild-type and mutants strains under various growth conditions, the strains were cultured in 11 different growth media which, apart from the control MM medium, have been reported by other workers to stimulate hyphal formation in C. albicans. The compositions of these media are listed in Supplementary Table 2. The $\mathrm{MM}^{-}$medium was the basic minimum mineral medium without any carbon or nitrogen sources. Other hyphae-inducing media were $\mathrm{MM}^{-}$ medium supplemented with L-arginine $(10 \mathrm{mM})$, L-glutamate $(10 \mathrm{mM})$, L-glutamine $(10 \mathrm{mM})$, L-proline $(10 \mathrm{mM})$, or $\mathrm{N}$-acetylglucosamine $(2.5 \mathrm{mM})$ at $\mathrm{pH}$ 6.5. The effect of ammonium $(10 \mathrm{mM})$ or urea $(10 \mathrm{mM})$ as the only nitrogen source on the growth of the different $C$. albicans strains at pH 6.5 was also investigated. The inoculated media were incubated in a rotary shaker at $37^{\circ} \mathrm{C}$ for $3 \mathrm{~h}$. The morphology of $C$. albicans cells in each growth medium was observed using a phase contrast microscope (DMR, Lecia).

\section{Growth characterisation and growth rates}

C. albicans mutant and wild-type strains were cultured in $30 \mathrm{~mL}$ of minimum mineral (MM) or $\mathrm{MM}^{-}$media supplemented with either L-arginine or L-proline. The growth yield of each culture was monitored by dry-weight measurements each hour until the stationary growth phase was reached. The growth rate was calculated by Eq. $1^{25}$, in which $\mu$ refers to the exponential growth rate; $B_{0}$ denotes biomass measured at the beginning of the exponential growth; $B$ is the biomass measured at the end of exponential growth; $t_{\mathrm{o}}$ is the time $(\mathrm{h})$ at the beginning of exponential growth; and $t$ is the time $(\mathrm{h})$ at the end of exponential growth.

$\mu=\frac{2.303 \times\left(\log (\mathrm{B})-\log \left(\mathrm{B}_{0}\right)\right)}{t-t_{0}}$

The growth rate formula

\section{Growth of yeast for metabolomic analysis}

C. albicans wild-type and mutant strains were cultured in $200 \mathrm{~mL}$ of YPD broth at $30^{\circ} \mathrm{C}$ in a rotary shaker overnight. The cells were collected by centrifugation at $2000 \mathrm{~g}$ for $5 \mathrm{~min}$ and washed in phosphate buffered saline. The cells were resuspended in $30 \mathrm{~mL}$ of culture media to an initial $\mathrm{OD}_{600}$ of 0.2 and incubated in a rotary shaker at $37^{\circ} \mathrm{C}$. There were 5 different culture media used in metabolomics and isotope labelling experiments: (1) Minimum mineral medium (MM medium) at $\mathrm{pH} 6.5$; (2) Arginine medium: $\mathrm{MM}^{-}$medium supplemented with L-arginine $(10 \mathrm{mM})$ at $\mathrm{pH} 6.5$; (3) ${ }^{13} \mathrm{C}_{6}$-arginine medium: $\mathrm{MM}^{-}$medium supplemented with $30 \%$ ${ }^{13} \mathrm{C}_{6}$-labelled $\left(30 \%{ }^{13} \mathrm{C}_{6}\right.$ and $\left.70 \%{ }^{12} \mathrm{C}_{6}\right)$ L-arginine $(10 \mathrm{mM})$ at $\mathrm{pH} 6.5$; (4) Proline medium: $\mathrm{MM}^{-}$medium supplemented with L-proline $(10 \mathrm{mM})$ at $\mathrm{pH}$ 6.5; (5) ${ }^{13} \mathrm{C}_{5}$-proline medium: $\mathrm{MM}^{-}$medium supplemented with $30 \%$ ${ }^{13} \mathrm{C}_{5}$-labelled $\left(30 \%{ }^{13} \mathrm{C}_{5}\right.$ and $\left.70 \%{ }^{12} \mathrm{C}_{5}\right)$ L-proline $(10 \mathrm{mM})$ at $\mathrm{pH} 6.5$.

\section{Sampling and quenching of microbial metabolism}

Five culture flasks $(30 \mathrm{~mL}$ ) of each $C$. albicans strain growing on $\mathrm{MM}$ medium, ${ }^{13} \mathrm{C}_{6}$-arginine medium, or ${ }^{13} \mathrm{C}_{5}$-proline medium were harvested at late exponential growth phase, while one flask of each culture growing on non-isotope labelled medium was harvested to estimate the natural abundance of ${ }^{13} \mathrm{C}$ in the samples. A portion $(5 \mathrm{~mL})$ of each culture was centrifuged at $2000 \times g$ for $5 \mathrm{~min}$ to remove $C$. albicans cells, and the supernatants were used for the analysis of extracellular metabolites. Another small portion $(2 \mathrm{~mL})$ of each culture was sampled and stored at $-80^{\circ} \mathrm{C}$ for ATP analysis. The remaining $23 \mathrm{~mL}$ of each culture was quickly filtered under vacuum (Air Cadet vacuum/pressure station, Thermo), immediately washed with cold saline solution $\left(9.5 \mathrm{~g} \mathrm{~L}^{-1} \mathrm{NaCl}\right.$ at $\left.1-2^{\circ} \mathrm{C}\right)$ and quenched in cold methanol/water $(1: 1 \mathrm{v} / \mathrm{v})$ at $-30^{\circ} \mathrm{C}$ as described by Smart et al. ${ }^{26}$.

\section{Sample preparation for metabolite analysis}

The internal standard $2,3,3,3-\mathrm{d}_{4}$-alanine $(0.3 \mu \mathrm{mol} / \mathrm{sample})$ was added to each sample before extraction. Freeze-thaw cycles and cold methanol/ water were used to extract the intracellular metabolites from the quenched cell pellets according to protocols described by Smart et al. ${ }^{26}$. The intracellular metabolite extracts and $2 \mathrm{~mL}$ of spent culture medium containing extracellular metabolites were freeze-dried (BenchTop $\mathrm{K}$ manifold freeze dryer, VirTis) prior chemical derivatisation.

\section{Chemical derivatisation of metabolites}

All freeze-dried samples were derivatised using the MCF method described by Smart et al. ${ }^{26}$. In brief, the freeze-dried samples were resuspended in $200 \mu \mathrm{L}$ sodium hydroxide $(1 \mathrm{M})$ and transferred to a silanised glass tube, then mixed with $167 \mu \mathrm{L}$ methanol and $34 \mu \mathrm{L}$ pyridine. The derivatisation was initiated by adding $20 \mu \mathrm{L}$ MCF followed by vigorously mixing for $30 \mathrm{~s}$, and then a further $20 \mu \mathrm{L}$ MCF was added followed by vigorously mixing for $30 \mathrm{~s}$. To separate MCF derivatives from the reaction mixture, $400 \mu \mathrm{L}$ chloroform was added and vigorously mixed for $10 \mathrm{~s}$ followed by the addition of $400 \mu \mathrm{L}$ sodium bicarbonate $(50 \mathrm{mM})$, and mixing for an additional $10 \mathrm{~s}$. The aqueous layer was discarded and the remaining water was removed with anhydrous sodium sulphate before samples were transferred to GC-MS vials.

\section{Gas chromatography-mass spectrometry (GC-MS) analysis}

The MCF derivatives were analysed in an Agilent GC7890 system coupled to a MSD5975 mass selective detector (El) operating at $70 \mathrm{eV}$. The gas column used for all analyses was a ZB-1701 GC capillary column $(30 \mathrm{~m} \times$ $250 \mu \mathrm{m}$ id $\times 0.15 \mu \mathrm{m}$ with $5 \mathrm{~m}$ guard column, Phenomenex). The analysis parameters were set according to Smart et al. $^{26}$. Samples were injected under pulsed splitless mode with the injector temperature at $290^{\circ} \mathrm{C}$. The helium gas flow through the GC-column was set at $1 \mathrm{~mL} \mathrm{~min}{ }^{-1}$. The interface temperature was set to $250^{\circ} \mathrm{C}$ and the quadrupole temperature was $200^{\circ} \mathrm{C}$. The mass spectrometry was operated in scan mode and started 
after 5.5 min with the mass range between 38 and 550 atomic mass units (amu) at 2.85 scans s $^{-1}$.

\section{Biomass quantification}

The cell debris collected after intracellular metabolite extraction was dried using a domestic microwave ( $250 \mathrm{~W}$ for $20 \mathrm{~min}$ ) and weighed in order to estimate the total biomass content (dry weight) of each sample.

\section{Data mining, data normalisation, and data analysis}

AMDIS software was employed to deconvolute GC-MS chromatograms and identify metabolites using our in-house MCF mass spectra library. The identifications were based on both MS spectrum of the derivatised metabolite and its respective chromatographic retention time. The relative abundance of identified metabolites was determined by ChemStation (Agilent) by using the GC base-peak value of a selected reference ion. These values were normalised by the biomass content in each sample as well as by the abundance of internal standard $\left(2,3,3,3-\mathrm{d}_{4}\right.$-alanine). A univariate analysis of variance (ANOVA) was used to determine whether the relative abundance of each identified metabolite was significantly different between wild-type and mutant strains under each growth condition. Our Pathway Activity Profiling (PAPi) algorithm ${ }^{27}$ was used to predict and compare the relative activity of different metabolic pathways between different $C$. albicans strains under the growth conditions tested. This programme is linked to the KEGG online database (http://www.kegg. com) and uses the number of metabolites identified from each pathway and their relative abundances to predict which metabolic pathway is likely to be active in the cell. The entire data mining, data normalisation, and pathway activity predictions were automated in our in-house $\mathrm{R}$ software package as described in Smart et al. ${ }^{26}$. Graphical representations of the results were produced by ggplot2 $\mathrm{R}$ packages ${ }^{28}$.

Calculating ${ }^{13} \mathrm{C}$-labelling enrichment in the detected metabolites The distribution of ${ }^{13} \mathrm{C}$-labelling from arginine or proline in the identified metabolite pool was determined by calculating the ratio of ${ }^{13} \mathrm{C}$ to ${ }^{12} \mathrm{C}$ in the major mass fragments. ${ }^{13} \mathrm{C}$-labelled metabolites were first identified based on their chromatographic retention time obtained from AMDIS. Together with the electron-impact fragmentation pattern of each identified MCF derivatised ${ }^{13} \mathrm{C}$-labelled metabolite, the degree of labelling was estimated based on the variation observed in the fragmentation pattern of ${ }^{13} \mathrm{C}$-labelled metabolites in comparison to their counterpart ${ }^{12} \mathrm{C}$ mass spectrum. Lastly, the total percentage of ${ }^{13} \mathrm{C}$ labelling was subtracted from the natural abundances of ${ }^{13} \mathrm{C}$ calculated from samples without added labelled substrates (Equation 2). This enabled identification of ${ }^{13} \mathrm{C}$ enrichment in the metabolites originating from the catabolism of ${ }^{13} \mathrm{C}$ labelled arginine and proline.

$$
\begin{aligned}
\% \text { of isotope labelling enrichment }= & \text { Enrichment }{ }^{13} C\left(\frac{(M+n) \text { ion mass height }}{(M) \text { ion mass height }} \times 100\right) \\
& - \text { Natural }{ }^{13} C\left(\frac{(M+n) \text { ion mass height }}{(M) \text { ion mass height }} \times 100\right)
\end{aligned}
$$

The percentage of isotope labelling enrichment formula. $\mathrm{M}$ is the ${ }^{12} \mathrm{C}$ ion of the metabolite; $n$ is a number of $\mathrm{m} / \mathrm{z}$ higher than $\mathrm{M} ; \mathrm{M}+n$ is the ${ }^{13} \mathrm{C}$ ion of the metabolite; Enrichment ${ }^{13} \mathrm{C}$ is ${ }^{13} \mathrm{C}$-labelled metabolites extracted from the cells grown in isotope enrichment medium; and Natural ${ }^{13} \mathrm{C}$ is the natural abundance of ${ }^{13} \mathrm{C}$ found in the cells grown in non-isotope enriched medium.

\section{Quantification of intracellular ATP pools}

The intracellular pool of ATP was determined by using the luminescence ATP detection assay ATPlite ${ }^{\text {TM }}$ (PerKinElmer). This assay measures light emission caused by the enzymatic reaction between ATP and luciferinluciferase. Medium without cells was used to determine the assay background signal for each medium. Three technical replicates of $100 \mu \mathrm{L}$ culture were taken from each culture flask sample prior to quenching cells in cold methanol/water. The cells were lysed and the total ATP content was quantified according to the manufacturer's instructions using ATP standard curves obtained from medium spiked with ATP standard solutions (one calibration curve for each medium). Light emission was measured using a bioluminescence plate reader (Envison ${ }^{\mathrm{TM}}$, PerkinElmer).
Quantification of intracellular $\mathrm{NAD}^{+} / \mathrm{NADH}$ and $\mathrm{NADP}^{+} / \mathrm{NADPH}$ pools

The intracellular pools of $\mathrm{NAD}^{+} / \mathrm{NADH}$ and $\mathrm{NADP}^{+} / \mathrm{NADPH}$ were quantified by using the Fluorimetric Amplite ${ }^{\mathrm{TM}} \mathrm{NAD}^{+} / \mathrm{NADH}$ and $\mathrm{NADP}^{+} / \mathrm{NADPH}$ assays (AAT Bioquest ${ }^{\circledR}$, Inc.). This assay measures light of wavelength of $590 \mathrm{~nm}$ emitted by an enzymatic reaction between fluorescent enzymes and cofactors when exposed to light with a wavelength of $540 \mathrm{~nm}$. The cells in three technical replicates of $1.25 \mathrm{ml}$ culture were collected and washed with PBS buffer (pH 7.4). The cells were lysed in cell lysis buffer with a Tissuelyser (Qiagen) at $30 \mathrm{~Hz}$ for $5 \mathrm{~min}$. The cofactors from cell lysates were measured according to the manufacturer instruction using cofactor standard curves. Fluorescence was measured using a fluorescence plate reader (Envison ${ }^{\mathrm{TM}}$, PerkinElmer) at $\mathrm{Ex} / \mathrm{Em}=540 / 590 \mathrm{~nm}$.

\section{Reporting Summary}

Further information on experimental design is available in the Nature Research Reporting Summary linked to this article.

\section{DATA AVAILABILITY}

The data that support the findings of this study are available from the corresponding author upon reasonable request.

\section{ACKNOWLEDGEMENTS}

We thank J.E. Upritchard, E. Lamping, and A.R. Holmes for technical assistance with gene knockout mutagenesis. K.P. Law for reviewd the manuscript. This work was supported by Faculty of Science Research Funding, University of Auckland and by a University of Auckland Doctoral Scholarship for Ting-Li Han.

\section{AUTHOR CONTRIBUTIONS}

S. B. and R. C. were involved in the research idea and design. S. G. and T. H. performed sample preparation, derivatisation, GC-MS analysis, and isotope labelling experiment. T. H. deleted the $G D H 2$ and $G D H 3$ genes. T. H. and S. G. analysed the data. T. H. wrote the manuscript. S. B. and R. C. revised the manuscript. All authors read and approved the final version of the manuscript.

\section{ADDITIONAL INFORMATION}

Supplementary information accompanies the paper on the npj Biofilms and Microbiomes website (https://doi.org/10.1038/s41522-019-0086-5).

Competing interests: We declare that the research was conducted in the absence of any financial or non-financial relationships that could be construed as a potential conflict of interest.

Publisher's note: Springer Nature remains neutral with regard to jurisdictional claims in published maps and institutional affiliations.

\section{REFERENCES}

1. Bendel, C. M., Hess, D. J., Garni, R. M., Henry-Stanley, M. \& Wells, C. L. Comparative virulence of Candida albicans yeast and filamentous forms in orally and intravenously inoculated mice. Crit. Care Med. 31, 501-507 (2003).

2. Saville, S. P., Lazzell, A. L., Monteagudo, C. \& Lopez-Ribot, J. L. Engineered control of cell morphology in vivo reveals distinct roles for yeast and filamentous forms of Candida albicans during infection. Eukaryot. Cell 2, 1053-1060 (2003).

3. Rooney, P. J. \& Klein, B. S. Linking fungal morphogenesis with virulence. Cell. Microbiol. 4, 127-137 (2002).

4. Lorenz, M. C., Bender, J. A. \& Fink, G. R. Transcriptional response of Candida albicans upon internalization by macrophages. Eukaryot. Cell 3, 1076-1087 (2004).

5. Rubin-Bejerano, I., Fraser, I., Grisafi, P. \& Fink, G. R. Phagocytosis by neutrophils induces an amino acid deprivation response in Saccharomyces cerevisiae and Candida albicans. Proc. Natl Acad. Sci. USA 100, 11007-11012 (2003).

6. Han, T., Cannon, R. D. \& Villas-Bôas, S. G. Metabolome analysis during the morphological transition of Candida albicans. Metabolomics 8, 1204-1217 (2012).

7. Han, T. L., Tumanov, S., Cannon, R. D. \& Villas-Boas, S. G. The metabolic response of Candida albicans to farnesol under hyphae-inducing conditions. FEMS Yeast Res. 12, 879-889 (2012). 
8. Han, T. L., Tumanov, S., Cannon, R. D. \& Villas-Boas, S. G. Metabolic response of Candida albicans to phenylethyl alcohol under hyphae-inducing conditions. PLoS ONE 8, e71364 (2013).

9. Villas-Bôas, S. G., Moxley, J. F., Akesson, M., Stephanopoulos, G. \& Nielsen, J. Highthroughput metabolic state analysis: the missing link in integrated functional genomics of yeasts. Biochem. J. 388, 669-677 (2005).

10. Han, T. L., Cannon, R. D. \& Villas-Bôas, S. G. The metabolic basis of Candida albicans morphogenesis and quorum sensing. Fungal Genet. Biol. 48, 747-763 (2011).

11. DeLuna, A., Avendano, A., Riego, L. \& Gonzalez, A. NADP-glutamate dehydrogenase isoenzymes of Saccharomyces cerevisiae. Purification, kinetic properties, and physiological roles. J. Biol. Chem. 276, 43775-43783 (2001).

12. Miller, S. M. \& Magasanik, B. Role of NAD-linked glutamate dehydrogenase in nitrogen metabolism in Saccharomyces cerevisiae. J. Bacteriol. 172, 4927-4935 (1990).

13. Avendano, A., Deluna, A., Olivera, H., Valenzuela, L. \& Gonzalez, A. GDH3 encodes a glutamate dehydrogenase isozyme, a previously unrecognized route for glutamate biosynthesis in Saccharomyces cerevisiae. J. Bacteriol. 179, 5594-5597 (1997).

14. Khale, A., Srinivasan, M. C. \& Deshpande, M. V. Significance of NADP/NAD glutamate dehydrogenase ratio in the dimorphic behavior of Benjaminiella poitrasii and its morphological mutants. J. Bacteriol. 174, 3723-3728 (1992).

15. Amin, A., Joshi, M. \& Deshpande, M. V. Morphology-associated expression of NADP-dependent glutamate dehydrogenases during yeast-mycelium transition of a dimorphic fungus Benjaminiella poitrasii. Antonie Van Leeuwenhoek J. Microbiol. 85, 327-334 (2004).

16. Peters, J. \& Sypherd, P. S. Morphology-associated expression of nicotinamide adenine dinucleotide-dependent glutamate dehydrogenase in Mucor racemosus. J. Bacteriol. 137, 1134-1139 (1979).

17. Sieg, A. G. \& Trotter, P. J. Differential contribution of the proline and glutamine pathways to glutamate biosynthesis and nitrogen assimilation in yeast lacking glutamate dehydrogenase. Microbiol. Res. 169, 709-716 (2014).

18. Miller, S. M. \& Magasanik, B. Role of the complex upstream region of the GDH2 gene in nitrogen regulation of the NAD-linked glutamate dehydrogenase in Saccharomyces cerevisiae. Mol. Cell Biol. 11, 6229-6247 (1991).

19. ter Schure, E. G., van Riel, N. A. \& Verrips, C. T. The role of ammonia metabolism in nitrogen catabolite repression in Saccharomyces cerevisiae. FEMS Microbiol. Rev 24, 67-83 (2000).

20. Reuß, O., Vik, Å., Kolter, R. \& Morschhäuser, J. The SAT1 flipper, an optimized tool for gene disruption in Candida albicans. Gene 341, 119-127 (2004).
21. Aon, J. C. \& Cortassa, S. Involvement of nitrogen metabolism in the triggering of ethanol fermentation in aerobic chemostat cultures of Saccharomyces cerevisiae. Metab. Eng. 3, 250-264 (2001).

22. Gillum, A. M., Tsay, E. Y. H. \& Kirsch, D. R. Isolation of the Candida albicans gene for orotidine-5'-phosphate decarboxylase by complementation of S. cerevisiae ura3 and E. coli pyrF mutations. Mol. Gen. Genet. 198, 179-182 (1984).

23. Noble, S. M. \& Johnson, A. D. Strains and strategies for large-scale gene deletion studies of the diploid human fungal pathogen Candida albicans. Eukaryot. Cell 4, 298-309 (2005).

24. Hernday, A. D., Noble, S. M., Mitrovich, Q. M. \& Johnson, A. D. Genetics and molecular biology in Candida albicans. Methods Enzymol 470, 737-758 (2010).

25. Villas-Bôas, S. G. et al. Phenotypic characterization of transposon-inserted mutants of Clostridium proteoclasticum B316T using extracellular metabolomics. J. Biotechnol. 134, 55-63 (2008).

26. Smart, K. F., Aggio, R. B. M., Van Houtte, J. R. \& Villas-Bôas, S. G. Analytical platform for metabolome analysis of microbial cells using methyl chloroformate derivatization followed by gas chromatography-mass spectrometry. Nat. Protoc. 5, 1709-1729 (2010).

27. Aggio, R. B. M., Ruggiero, K. \& Villas-Bôas, S. G. Pathway activity profiling (papi): from the metabolite profile to the metabolic pathway activity. Bioinformatics 26, 2969-2976 (2010).

28. Wickham, H. Ggplot: Elegant Graphics for Data Analysis (Springer, New York, 2009).

(c) Open Access This article is licensed under a Creative Commons Attribution 4.0 International License, which permits use, sharing, adaptation, distribution and reproduction in any medium or format, as long as you give appropriate credit to the original author(s) and the source, provide a link to the Creative Commons license, and indicate if changes were made. The images or other third party material in this article are included in the article's Creative Commons license, unless indicated otherwise in a credit line to the material. If material is not included in the article's Creative Commons license and your intended use is not permitted by statutory regulation or exceeds the permitted use, you will need to obtain permission directly from the copyright holder. To view a copy of this license, visit http://creativecommons. org/licenses/by/4.0/.

(c) The Author(s) 2019 